\title{
Conditional Moment Closure Modelling for HCCI with Temperature Inhomogeneities
}

\author{
Fatemeh Salehi ${ }^{\mathrm{a}, \mathrm{b}}$, Mohsen Talei ${ }^{\mathrm{c}}$ Evatt R. Hawkes ${ }^{\mathrm{b}, \mathrm{d}}$, \\ Ankit Bhagatwala ${ }^{\mathrm{e}}$, Jacqueline H. Chen ${ }^{\mathrm{e}}$, Chun Sang Yoo ${ }^{\mathrm{f}}$, Sanghoon Kook ${ }^{\mathrm{d}}$ \\ ${ }^{a}$ School of Aerospace, Mechatronic and Mechanical Engineering, \\ The University of Sydney, NSW 2006, Australia \\ b School of Photovoltaic and Renewable Energy Engineering, \\ University of New South Wales, Sydney, 2052, Australia \\ ${ }^{c}$ Department of Mechanical Engineering, \\ University of Melbourne, Parkville, VIC 3010, Australia \\ d School of Mechanical and Manufacturing Engineering, \\ University of New South Wales, Sydney, 2052, Australia \\ e Combustion Research Facility, \\ Sandia National Laboratories,Livermore, CA 94550, United States \\ ${ }^{\mathrm{f}}$ Ulsan National Institute of Science and Technology, \\ Ulsan 689-798, Republic of Korea
}

Corresponding author:

Fatemeh Salehi

School of Aerospace, Mechatronic and Mechanical Engineering,

The University of Sydney, NSW 2006, Australia

Phone: +61(4)2360949

Email: fatemeh.salehi@sydney.edu.au

Preferred colloquium topic area:

IC Engine Combustion

Total length of paper:

$(6.89$ pages $* 900)=\mathbf{6 2 0 1}(\operatorname{method} 2)$

Main text and references: $(5.8$ pages $* 900)=5220$

Tables: $(26) * 4.4=115$

Figures and captions: $(52+60+122) * 2.2+(40+40) * 4.4=866$ 


\title{
Doubly Conditional Moment Closure Modelling for HCCI with Temperature Inhomogeneities
}

\author{
Fatemeh Salehi ${ }^{\mathrm{a}, \mathrm{b}, *}$, Mohsen Talei $^{\mathrm{c}}$, Evatt R. Hawkes ${ }^{\mathrm{b}, \mathrm{d}}$, Ankit Bhagatwala ${ }^{\mathrm{e}}$, Jacqueline H. \\ Chen $^{\mathrm{e}}$, Chun Sang Yoo ${ }^{\mathrm{f}}$, Sanghoon Kook ${ }^{\mathrm{d}}$ \\ ${ }^{a}$ School of Aerospace, Mechatronic and Mechanical Engineering, The University of Sydney, NSW 2006, \\ Australia \\ ${ }^{b}$ School of Photovoltaic and Renewable Energy Engineering, The University of New South Wales, Sydney, \\ NSW 2052, Australia \\ ${ }^{c}$ Department of Mechanical Engineering, University of Melbourne, Parkville, VIC 3010, Australia \\ ${ }^{d}$ School of Mechanical and Manufacturing Engineering, The University of New South Wales, Sydney, NSW \\ 2052, Australia \\ ${ }^{e}$ Combustion Research Facility, Sandia National Laboratories, Livermore, CA 94550, United States \\ ${ }^{f}$ School of Mechanical and Nuclear Engineering, \\ Ulsan National Institute of Science and Technology, Ulsan 689-798, Republic of Korea
}

\begin{abstract}
This paper presents a doubly conditional moment closure (DCMC) as an a posteriori predictive modelling tool for ignition of mixtures with large thermal stratification in homogeneous charge compression ignition (HCCI) conditions. Double conditioning is applied on enthalpy and its dissipation rate. The performance of the DCMC model is evaluated using a number of previously reported direct numerical simulations (DNSs) with various fuels. The DNSs modelled ignition of various lean homogeneous mixtures with a high level of temperature inhomogeneities. The selected cases exhibit a prevalence of deflagration mode of combustion as opposed to a spontaneous ignition-front mode, which has proven challenging for previous singly CMC. In all simulations, DCMC solver is run in a stand-alone mode with certain terms, such as the probability density functions of enthalpy and dissipation rate, being provided using the DNS input. The DCMC results are in a very good agreement with the DNS data, and are significantly improved compared with a singly conditional moment closure. A set of a posteriori DNS-DCMC tests is also performed to demonstrate importance of various terms in the doubly CMC equations. These tests first reveal that the effects of the cross dissipation and sources of enthalpy and dissipation rate (which lead to convective terms in conditional space) are insignificant and these terms can be safely neglected from the DCMC equations. The significance of this result is that the main unclosed models that would be needed
\end{abstract}

\footnotetext{
${ }^{*}$ Corresponding author

Email address: fatemeh.salehi@sydney.edu.au (Fatemeh Salehi)
} 
for satisfactory results in a practical simulation of an engine would be the joint probably density function of enthalpy and its dissipation rate and the dissipation rate of dissipation rate.

Keywords:

Conditional moment closure; thermal stratification; autoignition; HCCI.

\section{Introduction}

Reducing formation of pollutants and optimising engine efficiency are key concerns in developing engine technologies. A possible solution, having potential for achieving high efficiency and low emissions, can be homogeneous charge compression ignition (HCCI). Using the HCCI concept nitrogen oxides $\left(\mathrm{NO}_{\mathrm{x}}\right)$ and soot formation are suppressed by keeping the flame temperature below the formation threshold via overall lean mixtures. However, lack of a means to control the combustion phasing, especially during transients, and the high rate of pressure rise at high load conditions pose significant technical challenges [1]. Hence, HCCI is still in the phase of laboratory research and has not seen commercial success yet. Nevertheless, HCCI could well be effective in particular operating regimes and combined with other strategies where HCCI is too challenging.

Thermal stratification in an HCCI engine cylinder can reduce the pressure rise rate under highload $[1,2]$. Effects of thermal stratification on the ignition process have been widely studied by direct numerical simulation (DNS) for various mixtures under HCCI conditions [3-8]. The DNS studies show that different combustion modes can be observed depending on the degree of thermal stratification [3-8]. Under low levels of thermal stratification, the spontaneous combustion mode is prevalent. However, under high thermal stratification conditions, gradients of ignition delay are sufficiently large to result in significant fraction of burning in premixed flames where conduction and diffusion effects become important, i.e. in a deflagrative mode of combustion.

In previous study [9], a model based on the first-order singly conditional moment closure (CMC) was developed to study HCCI combustion. The basic idea behind the CMC approach is that errors in the evaluation of the unclosed reaction source term can be significantly reduced if its evaluation is conditioned on another variable upon which the reaction rate mainly depends [10]. The CMC model for HCCI was evaluated using various sets of DNS data $[4,5]$ with various levels of temperature fluctuations. It was found that the first-order singly CMC model cannot predict the ignition process as well in conditions of large thermal stratification compared with low and medium stratification levels. In the former conditions, the deflagration mode is dominant and there is a strong correlation between dissipation fluctuations and conditional fluctuations of scalars 
(e.g. temperature and radical mass fractions) which are not considered in the first-order singly CMC model $[9,11]$.

This paper makes an original contribution towards advancing combustion modelling under HCCI conditions, aiming to improve the performance at high stratification levels. There are various options for achieving this including higher order closures [10, 12-16] or conditioning on multiple variables $[17,18]$. For example, a second-order closure is a possible extension of the first-order singly closure [10]. In second-order closures, using Taylor expansions, effects of species-species and temperature-species correlations are accounted for in the conditional reaction rates. However, an a priori study [19] revealed that the second-order conditional moment closure was not a promising solution for large stratifications. Second-order CMC is also computationally expensive, particularly for more realistic hydrocarbon fuels involving a chemistry mechanism with a large number of species. Alternatively, double conditioning of the reactive scalar transport equations can be used to improve the first-order singly conditional closure prediction [17, 18, 20-22]. By choosing a suitable second conditioning variable, the fluctuations around the doubly conditioned mean can be reduced in comparison with the singly conditioned case and then the first-order closure of the conditional chemical source term remains valid in which doubly conditioned reaction rate is a function of doubly conditioned species mass fraction and temperature.

An extension of the first-order singly CMC to include a second conditioning variable was initially proposed by Bilger [20] and it has been investigated for non-premixed flames in Refs. [17, 18, 21-23]. In earlier studies for non-premixed flames, mixture fraction was considered as the first conditioning variable while several second conditioning variables such as dissipation rate [21], sensible enthalpy $[17,18]$ and a normalised mixture fraction-dependent temperature [22] have been proposed with varying degrees of success and complexity. The doubly CMC model in the context of premixed combustion has not been reported in the literature to the best of our knowledge.

For autoignition of a thermally stratified but compositionally homogeneous mixture, as considered here, total enthalpy is a natural choice of conditioning variable, as noted in our previous studies $[9,11]$. Total enthalpy is attractive not only because it maps to temperature and thus the autoignition delay, but importantly because its source term $\left(\frac{D p}{D t}\right)$ is homogeneous in space to within acoustic fluctuations.

In choosing the second conditioning variable, it is noted that the focus of this study is on thermal stratification cases where the deflagration mode is found to be important. A previous assessment of the conditional variance equations [11] revealed that the correlation between dissipation rate fluctu- 
ations and conditional mass fraction fluctuations played an important role to generate conditional mass fraction fluctuations in high temperature inhomogeneity conditions. This motivates the use of the scalar dissipation rate as a second conditioning variable to improve the CMC prediction for large temperature stratification conditions. More discussion of this choice is given later.

The objective of this paper is to evaluate the potential of DCMC for modelling stratified ignitions using DNS. DCMC requires some model inputs that would normally be provided by a computational fluid dynamics solver, namely the joint probability density functions of enthalpy and its dissipation rate, enthalpy and dissipation source terms, etc. In order to focus this paper exclusively on issues surrounding the double conditioning, these terms are provided in this study directly from the DNS. With these inputs the DCMC is run a posteriori and compared with the DNS data and with first-order singly CMC results.

The paper is organised as follows. Section 2 and Section 3 outline the main details of the CMC and DCMC respectively, Section 4 presents the details of the previously reported DNS cases used in the analysis, section 5 presents the key findings while section 6 concludes.

\section{Singly conditional moment closure (CMC)}

First-order singly CMC equations for ignition in thermally stratified mixtures are briefly described here. Further details can be found in Ref. [9]. A normalised scalar variable is defined as,

$$
\theta(x, t)=\frac{h(x, t)-h_{\min }(t)}{h_{\max }(t)-h_{\min }(t)},
$$

where $h$ is the enthalpy and $h_{\min }$ and $h_{\max }$ correspond to the minimum and maximum enthalpies in the computational domain, respectively. The singly conditional Favre-averaged mass fraction of species $\alpha, Q_{\alpha}^{I}$, at time $t$ and location $x$ is defined as

$$
Q_{\alpha}^{I}(\xi ; x, t)=\left\langle\left. Y_{\alpha}\right|_{\xi}\right\rangle=\frac{\overline{\left.\rho Y_{\alpha}(x, t)\right|_{\theta=\xi}}}{\overline{\left.\rho\right|_{\theta=\xi}}},
$$

where $\xi$ is the sample space variable for normalised enthalpy, $\rho$ is the density and $Y_{\alpha}$ is the species mass fraction of species $\alpha$. The CMC transport equations can be obtained with this definition [9]:

$$
\frac{\partial Q_{\alpha}^{I}}{\partial t}=-\left\langle\left. S_{\theta}\right|_{\xi}\right\rangle \frac{\partial Q_{\alpha}^{I}}{\partial \xi}+\left\langle\left. N_{\theta}\right|_{\xi}\right\rangle \frac{\partial^{2} Q_{\alpha}^{I}}{\partial \xi^{2}}+\frac{\overline{\left.\dot{\omega}_{\alpha}\right|_{\xi}}}{\overline{\left.\rho\right|_{\xi}}},
$$

where $N_{\theta}$, the scalar dissipation rate, is $D \frac{\partial \theta}{\partial x_{j}} \frac{\partial \theta}{\partial x_{j}}$, and $\dot{\omega}_{\alpha}$ is the chemical reaction rate. The convective source, $S_{\theta}$, results from the source term of normalised enthalpy that is defined as: 


$$
S_{\theta}=\frac{1}{\Delta h}\left(\frac{1}{\rho} \frac{D p}{D t}-(1-\theta) \frac{\partial h_{\min }}{\partial t}-\theta \frac{\partial h_{\max }}{\partial t}\right)
$$

where $p$ is the pressure and $\Delta h$ is the difference between the maximum and the minimum enthalpies in the domain. The cylinder charge is assumed to be homogeneous in terms of conditional statistics (i.e. conditioning on enthalpy is sufficient to collapse any spatial structure inside the engine), and hence, the spatial terms in physical space are ignored. Under the primary first-order CMC assumption, the conditional fluctuations are small enough such that the singly conditional reaction rates can be obtained as

$$
\overline{\left.\dot{\omega}_{\alpha}\right|_{\xi}}=f\left(Q_{\alpha}^{I},\left\langle\left. T\right|_{\xi}\right\rangle, \overline{\left.p\right|_{\xi}}\right)
$$

where $T$ is the temperature.

\section{Doubly conditional moment closure (DCMC)}

A transport equation for the scalar dissipation rate can be readily derived using the method presented in Ref. [24]:

$$
\rho \frac{\partial \chi}{\partial t}=-\rho v_{j} \frac{\partial \chi}{\partial x_{j}}+\rho \frac{\partial}{\partial x_{j}}\left(D \frac{\partial \chi}{\partial x_{j}}\right)+\rho S_{\chi}
$$

where $\chi \equiv N_{\theta}=D \frac{\partial \theta}{\partial x_{j}} \frac{\partial \theta}{\partial x_{j}}$. The source term, $S_{\chi}$, on the right hand side of Eq. (5) is:

$$
S_{\chi}=-2 \chi \frac{\partial v_{j}}{\partial x_{j}}-2 \chi \frac{1}{\Delta h} \frac{\partial \Delta h}{\partial t}-2 D^{2} \frac{\partial^{2} \theta}{\partial x_{j}^{2}} \frac{\partial^{2} \theta}{\partial x_{j}^{2}} .
$$

A normalised scalar dissipation rate is defined as:

$$
\phi(x, t)=\frac{\chi(x, t)-\chi_{\min }(t)}{\chi_{\max }(t)-\chi_{\min }(t)}
$$

where the scalars $\chi_{\min }$ and $\chi_{\max }$ refer to the minimum and maximum scalar dissipation rates in the domain.

The doubly conditional mean of species mass fraction is defined as

$$
Q_{\alpha}^{I I}(\xi ; \eta ; x, t)=\left\langle\left. Y_{\alpha}\right|_{\xi, \eta}\right\rangle=\frac{\overline{\left.\rho Y_{\alpha}(x, t)\right|_{\theta=\xi, \phi=\eta}}}{\overline{\left.\rho\right|_{\xi, \eta}}},
$$

where $\eta$ is the sample space variable for normalised dissipation rate, $\phi$. With this definition, doubly conditional moment closure equations can be obtained using the transport equation for scalar dissipation rate, Eq. (5): 


$$
\begin{aligned}
\frac{\partial Q_{\alpha}^{I I}}{\partial t} & =-\underbrace{\left\langle\left. S_{\theta}\right|_{\xi, \eta}\right\rangle \frac{\partial Q_{\alpha}^{I I}}{\partial \xi}}_{\mathrm{T} 1}-\underbrace{\left\langle\left. S_{\phi}\right|_{\xi, \eta}\right\rangle \frac{\partial Q_{\alpha}^{I I}}{\partial \eta}}_{\mathrm{T} 2} \\
& +\underbrace{\left\langle\left. N_{\theta}\right|_{\xi, \eta}\right\rangle \frac{\partial^{2} Q_{\alpha}^{I I}}{\partial \xi^{2}}}_{\mathrm{T} 3}+\underbrace{\left\langle\left. N_{\phi}\right|_{\xi, \eta}\right\rangle \frac{\partial^{2} Q_{\alpha}^{I I}}{\partial \eta^{2}}}_{\mathrm{T} 4} \\
& +2 \underbrace{2\left\langle\left. N_{\theta, \phi}\right|_{\xi, \eta}\right\rangle \frac{\partial^{2} Q_{\alpha}^{I I}}{\partial \xi \partial \eta}}_{\mathrm{T} 5} \\
& +\underbrace{\frac{\dot{\omega}_{\left.\alpha\right|_{\xi, \eta}}}{\left.\bar{\rho}\right|_{\xi, \eta}}}_{\mathrm{T} 6},
\end{aligned}
$$

where $N_{\phi}$, the dissipation of dissipation, is $D \frac{\partial \phi}{\partial x_{j}} \frac{\partial \phi}{\partial x_{j}}$ and $N_{\theta, \phi}$, the cross dissipation of $\theta$ and $\phi$, is $D \frac{\partial \theta}{\partial x_{j}} \frac{\partial \phi}{\partial x_{j}}$. The term $S_{\phi}$ results from the source term of normalised dissipation which can obtained using Eqs. (5) and (6):

$$
\begin{aligned}
S_{\phi} & =\frac{1}{\Delta \chi}\left(-\phi \frac{\partial \chi_{\max }}{\partial t}\right. \\
& -(1-\phi) \frac{\partial \chi_{\min }}{\partial t} \\
& -2\left(\Delta \chi \phi+\chi_{\min }\right)\left(\frac{\partial v_{j}}{\partial x_{j}}+\frac{1}{\Delta h} \frac{\partial \Delta h}{\partial t}\right) \\
& \left.-2 D^{2} \frac{\partial^{2} \theta}{\partial x_{j}^{2}} \frac{\partial^{2} \theta}{\partial x_{j}^{2}}\right) .
\end{aligned}
$$

Note that to derive Eq. (8), the primary CMC closure hypothesis was applied and second order conditional correlations between the source-terms of the conditioning variables and the scalars were neglected, consistent with a first order closure approach. Similar to the singly CMC model, all transport terms in physical space in Eq. (8) are ignored. It is assumed that the conditional fluctuations around a doubly conditional mean are small and hence doubly conditional reaction rates can be obtained using the doubly conditional species mass fraction and temperature:

$$
\overline{\left.\dot{\omega}_{\alpha}\right|_{\xi, \eta}}=f\left(Q_{\alpha}^{I I},\left\langle\left. T\right|_{\xi, \eta}\right\rangle, \overline{\left.p\right|_{\xi, \eta}}\right) .
$$

The conditioned quantities can be obtained from doubly conditioned quantities by integration across $\eta$-space, 


$$
\begin{aligned}
\left\langle\left. Y_{\alpha}\right|_{\xi}\right\rangle & =\frac{1}{P(\xi)} \int\left\langle\left. Y_{\alpha}\right|_{\xi, \eta}\right\rangle P(\xi, \eta) d \eta \\
\overline{\left.\dot{\omega}_{\alpha}\right|_{\xi}} & =\frac{1}{P(\xi)} \int \overline{\left.\dot{\omega}_{\alpha}\right|_{\xi, \eta}} P(\xi, \eta) d \eta
\end{aligned}
$$

where $P(\xi, \eta)$ is the joint probably density function (PDF) of the normalised enthalpy, $\theta$, and the normalised scalar dissipation, $\phi$, whereas $P(\xi)$ is the PDF of normalised enthalpy, $\theta$.

\section{Test cases studied and numerical methods}

Four previously reported two-dimensional DNS cases were used here to examine the performance of the DCMC for a range of fuels having different autoignition characteristics. The DNS data sets modelled the ignition of a lean ethanol/air [25], $n$-heptane/air [4] and iso-octane/air [5] mixtures with large thermal stratifications in a constant volume. All DNS cases were initialised with a uniform mixture composition field. The physical domain in the DNS studies was $3.2 \mathrm{~mm}$ square for the $n$-heptane and iso-octane cases, whereas it was $3.6 \mathrm{~mm}$ square for the ethanol case. Periodic boundary conditions were applied in both directions in all cases. The main parameters of the DNS cases are shown in Table 1 . The initial pressure is donated $p_{0}$; the equivalence ratio $\lambda$; the mean temperature $T_{0}$; the temperature fluctuation root mean square (RMS) $T^{\prime}$; the turbulence velocity fluctuation $u^{\prime}$ and the most energetic turbulent length scale $l_{e}$. The ethanol and iso-octane cases feature single-stage ignition, whereas the $n$-heptane cases features two-stage ignition. The aim of the case selection is to evaluate the DCMC model for various compositions with different levels of temperature inhomogeneities, where the deflagration mode is prevalent in all cases. A dilution level of $33 \%$ mole fraction of simulated exhaust gas recirculation (EGR) was only employed in the ethanol case. As an example, the initial temperature field for case ethanol-5 is shown in Fig. 1. The CMC simulations employ the same chemistry mechanisms as the DNSs including a 29-, 58and 99-species reduced mechanism for ethanol/air [25], n-heptane/air [4] and iso-octane/air [5], respectively.

The open source $\mathrm{C}++$ code known as OpenFOAM was employed to implement both singly and doubly CMC models. Since the focus of this study is to investigate the fundamental closure assumptions, and not on other required closures such as for turbulence stresses, etc., the singlyand doubly-CMC models were coupled with the DNS database to provide some inputs that would normally require modelling. Therefore, in the single CMC model the coefficients in Eq. (2) including 
the conditional dissipation rate, $\left\langle\left. N_{\theta}\right|_{\xi}\right\rangle$ and the convective velocity in conditional space, $\left\langle\left. S_{\theta}\right|_{\xi}\right\rangle$, were directly taken from the DNS data. Similarly, coefficients in Eq. (8) for the DCMC model consisting of the doubly conditional dissipation rate, $\left\langle\left. N_{\theta}\right|_{\xi, \eta}\right\rangle$, doubly conditional dissipation of dissipation, $\left\langle\left. N_{\phi}\right|_{\xi, \eta}\right\rangle$, doubly conditional cross dissipation rate, $\left\langle\left. N_{\theta, \phi}\right|_{\xi, \eta}\right\rangle$, and convective sources, $\left\langle\left. S_{\theta}\right|_{\xi, \eta}\right\rangle$ and $\left\langle\left. S_{\phi}\right|_{\xi, \eta}\right\rangle$, were provided using the DNS data. The PDF of the normalised enthalpy, $P(\xi)$, and joint PDF of the normalised enthalpy and normalised scalar dissipation, $P(\xi, \eta)$, were also obtained from the DNS data. Pressure, $h_{\min }$ and $h_{\max }$, are also provided by the DNS. As a result, the CMC solvers were run in a stand-alone mode using the DNS input.

It should be noted that when the DCMC model is coupled with a practical CFD package, models of the PDFs which account for the unresolved turbulent fluctuations would need to be provided. Since the enthalpy source term does not fluctuate significantly in space, it is expected to behave similarly to a passive scalar, for which established models are already available. For the enthalpy PDF, standard models, such as a two-parameter presumed beta PDF are applicable [9]. The conditional PDF of a passive scalars dissipation rate is known to have a nearly lognormal distribution [26], which requires input of the mean and variance. The conditional mean is well approximated by amplitude mapping closure [27] while the variance is a function of the mean and Reynolds number [26]. The joint PDF of enthalpy and its dissipation rate can be readily obtained using the enthalpy PDF and the conditional PDF of dissipation rate. Testing these models is outside of the scope of the present work and is a topic of our future research.

A three-step fractional method was employed to advance the solution of both singly and doubly CMC equations, Eqs. (2) and (8), respectively, in time. The first and second-order derivatives were discretised using the first-order upwind and second-order central difference schemes, respectively. A uniform mesh of 40 bins are considered in both normalised enthalpy, $\xi$, and normalised dissipation, $\eta$. The CMC domains were initialised by sub-sampling the DNS and subsequently evolve independently with the previously specified inputs and reaction rates computed using Eq. (4) and Eq. (10), for the single and doubly CMC, respectively.

\section{Results}

Figure 2 presents the temporal evolution of the mean heat release rate from the DNS and that computed with both singly and doubly CMC models for all cases. Time is normalised by the ignition delay time, $\tau_{i g}$, in each case. The singly CMC model is first discussed. This model overpredicts the mean heat release rates at early stages of the ignition process while the model under predicts 
Table 1: Physical parameters of the DNS and CMC cases.

\begin{tabular}{ccccccc}
\hline Case & $p_{0}(\mathrm{~atm})$ & $\lambda$ & $T_{0}(\mathrm{~K})$ & $T^{\prime}(\mathrm{K})$ & $u^{\prime}(\mathrm{m} / \mathrm{s})$ & $l_{e}(\mathrm{~mm})$ \\
\hline ethanol-5 & 44.4 & 0.4 & 924 & 40 & 0.61 & 0.65 \\
octane-3 & 20.0 & 0.3 & 1035 & 60 & 0.5 & 1.25 \\
heptane-5 & 40.0 & 0.3 & 850 & 100 & 0.5 & 1.24 \\
heptane-9 & 40.0 & 0.3 & 934 & 100 & 0.5 & 1.24 \\
\hline
\end{tabular}

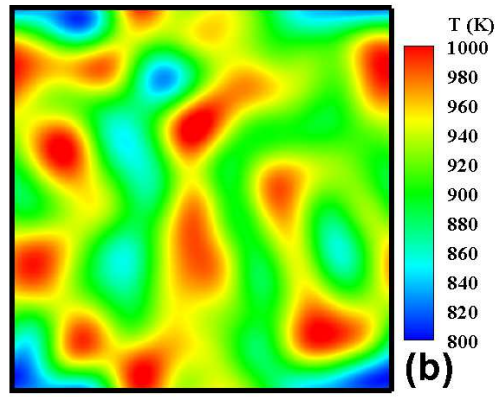

Figure 1: Initial temperature for case ethanol-5 with $T^{\prime}=40 \mathrm{~K}$.

the mean heat release close to the main ignition delay time in all cases. As a result, the first-order model leads to advanced ignition with a lower peak heat release. As discussed previously [9, 11], for a high level of temperature inhomogeneities, the conditional fluctuations are large and therefore the singly CMC cannot predict the ignition process very well. This is due to the dominance of in these cases deflagration, which can propagate independently of ignition delay gradients and thus generate conditional fluctuations, as demonstrated in the original DNS studies [4, 5, 25].
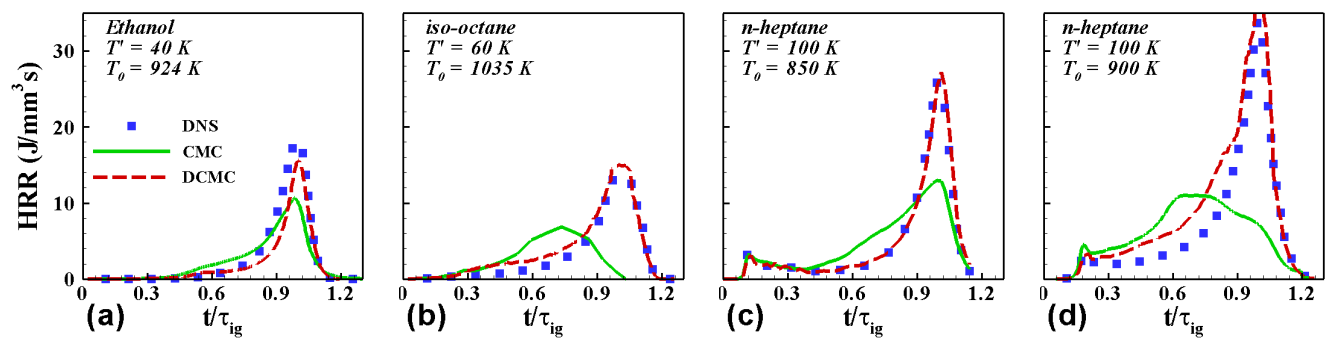

Figure 2: Modelling comparison; temporal evaluation of mean heat release rate in all cases.

To motivate the choice of scalar dissipation as the second conditioning variable, Figure 3 shows scatter plots of the local conditional fluctuation of fuel mass fraction, $Y_{\alpha}-\left\langle\left. Y_{\alpha}\right|_{\xi}\right\rangle$, versus normalised 
enthalpy at a time of $0.45<t / \tau_{i g}<0.6$. The conditional fluctuations are normalised with the initial fuel mass fraction for each case and they are colour-coded by $\log \left(N_{\theta}\right)$. As can be seen, the conditional fluctuations are significant, which is a result of the increased importance of deflagrative modes of combustion in these cases. It can be seen that the conditional fluctuations are strongly correlated with the scalar dissipation rate in all cases. The negative conditional fluctuations, i.e. more advanced combustion, of the fuel mass fraction are frequently coincident with high dissipation structures, whereas the opposite is true for positive conditional fluctuations. As discussed in earlier work [11], this correlation arises because larger dissipation rates imply faster mixing between already burning regions and as yet unburned regions, accelerating reaction progress in the latter. This demonstrates that the use of the scalar dissipation rate as a second conditioning variable can distinguish regions of different levels of reaction progress.
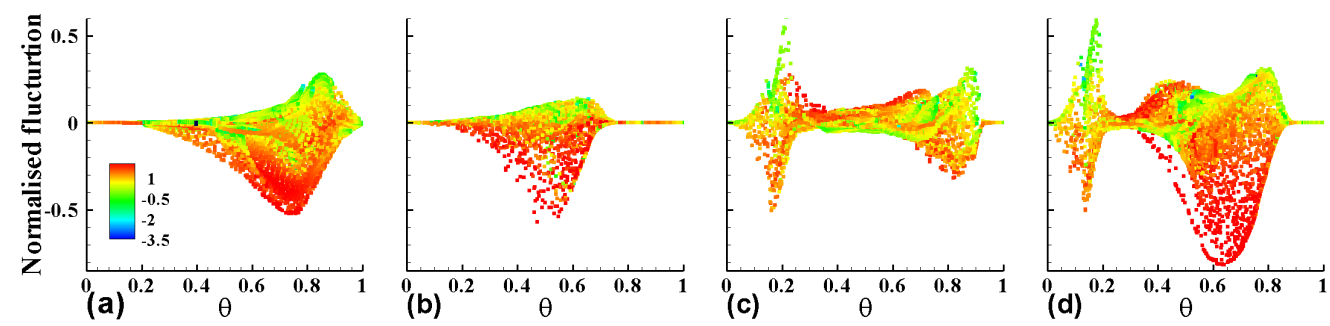

Figure 3: Scatter plots of normalised $Y_{\alpha}-\left\langle\left. Y_{\alpha}\right|_{\xi}\right\rangle$ colour by $\log \left(N_{\theta}\right)$ versus normalised enthalpy for cases a) ethanol-5, b) octane-3, c) heptane-5, and d) heptane-9.

To verify the implementation of the doubly conditional equation, and to verify that neglected terms are not significant, the overall effects of the right and left hand sides of Eq. (8) for fuel mass fraction in the ethanol case with $T^{\prime}=40 \mathrm{~K}$ are shown in Fig. 4. In this test, all terms, including the conditionally averaged reaction rate terms, are directly provided by the DNS. It can be seen that the doubly conditional species mass fraction is closed. This verifies the implementation and confirms that the primary closure hypothesis that is needed to derive the doubly CMC equations is correct and that conditional correlations between the conditioning variable source term and scalars are negligible. It should be noted that the small observed discrepancy is likely associated with numerical errors in calculating the second derivatives and the mixed derivative of doubly conditional fuel mass fraction. Since the doubly conditional mean contains statistical noise, some errors arise in taking derivatives.

Simulations using the DCMC model are then performed and the results are presented in Fig. 2. It can be seen that the DCMC model significantly improves the prediction of the mean heat 


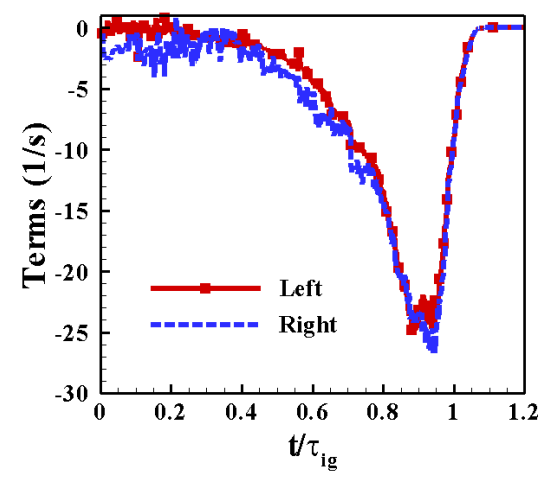

Figure 4: Comparison of the left and right sides of doubly CMC equations, Eq. (8), for fuel mass fraction in case ethanol-5 with $T^{\prime}=40 \mathrm{~K}$.

release rate compared to the first-order CMC model in all cases. The ignition delay time and the peak heat release rate are predicted very well in case ethanol-5 and heptane-5. It can be seen that the ignition process is slightly ahead of the DNS at an early phase in cases heptane-9 and octane-3, and the peak of heat release rate is slightly over-predicted but the agreement is certainly satisfactory and considerable better than the singly CMC. It can been also seen that the DCMC agrees better with the DNS in case heptane-5 compared to case heptane-9. Our investigation has not uncovered a clear explanation for this observation, however this better agreement was expected as the singly CMC prediction also showed a better agreement for this case. It is found that the level of agreement between DNS and DCMC results for the main species and the temperature is also similar to the heat release rates and the results are presented in the supplementary material to this paper.

Modelling some of the terms in Eq. (8) that are provided by the DNS here would in a practical setting be required to be provided by quantities available from large scales (i.e. from a computational fluid dynamics package). Some of these terms could be challenging, notably the source term of dissipation rate, the cross dissipation rate, and the dissipation of dissipation. Therefore, to shed more light on effects of different terms in the doubly CMC equations, Eq. (8), some a posteriori tests are defined here. The model is run with some of the terms set to zero in order to determine whether those terms are important. The results for the mean heat release rate for these tests in comparison with the full model are shown in Fig. 5 for both cases ethanol-5 and octane-3. In the first test, the convective type terms ( $\mathrm{T} 1$ and $\mathrm{T} 2$ ), which arise due to sources of enthalpy and its dissipation are set to zero. Figure 5 shows that the results in comparison with the full model (DCMC) are almost identical for both cases, showing that these terms are not important. 
This might be expected since the conditioning variables are normalised here. In the next test, the cross dissipation term (T5) is additionally eliminated. The results in Fig. 5 show that this term is also insignificant. This is consistent with an earlier observation for a non-premixed flame in Ref. [21]. Finally, in test 3, the mixing in dissipation space is also eliminated, leaving only mixing in enthalpy space (conditioned on dissipation) and the chemical reaction term remaining. The results here show an $8 \%$ and a $12 \%$ under-prediction of the peak heat release rate in cases ethanol-5 and octane-3, respectively. However, overall the agreement is acceptable and significantly better than for the singly CMC in both cases. The significance of these findings is discussed shortly in the conclusions.
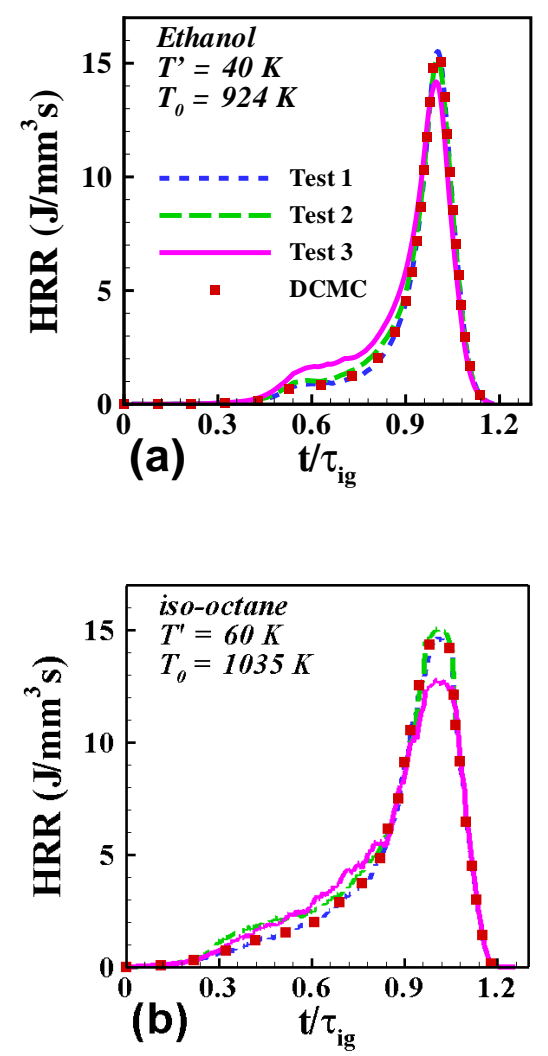

Figure 5: Evaluation of the different terms in DCMC equations, Eq. (8), for cases a) ethanol-5 with $T^{\prime}=40$ $\mathrm{K}$ and b) octane- 3 with $T^{\prime}=60 \mathrm{~K}$. Test 1 , mixing in both $\theta$ - and $\phi$ - spaces and their cross; Test 2 , mixing in both $\theta$ - and $\phi$ - spaces; Test 3 , only mixing in $\theta$-space. 


\section{Conclusion}

An a posteriori study of doubly conditional moment closure (DCMC) model, with conditioning on normalised total enthalpy and its dissipation rate, was presented for application to thermally stratified ignitions. Four two-dimensional DNS cases were selected to study the DCMC model. The DNS cases modelled ignition of various mixtures with high levels of temperature inhomogeneities, and with fuels with differing ignition characteristics (single-stage and two-stage). The model prediction for the ignition process was evaluated against the DNSs and the results obtained using the first-order singly CMC model. In both DCMC and first-order singly CMC simulations, some required input were provided using the DNS. The DCMC model presented a significant improvement in predicting the ignition process compared to the singly CMC in all cases. The doubly CMC model showed an excellent agreement with the DNS for cases ethanol-5 and heptane-5. A slightly advanced ignition at an early phase of combustion was observed in cases heptane- 9 and octane-3 though agreement was certainly acceptable and significantly better compared to the singly CMC model.

To demonstrate the importance of different terms in doubly CMC equations, a set of a posteriori tests was defined and applied to cases ethanol-5 and octane-3. A significant finding was that when all of terms in the equation except the mixing in enthalpy space were neglected, satisfactory results were still achieved. As the doubly conditional dissipation rate is obviously just dissipation rate itself, the conditional mixing term is already closed and the only 'model' that a CFD solver would need to provide in this simplified DCMC would be the joint PDF of enthalpy and its dissipation rate. As a result, this simplified version of the model becomes quite similar to an ensemble of interactive flamelets which each experience a different dissipation rate [28]. However, this approach did not give perfect results, under-predicting the peak heat release by $8-12 \%$. Less satisfactory performance should be expected for higher levels of fluctuations with this approach.

However, better results were obtained by additionally considering mixing in dissipation space, which would require only an additional model for the doubly conditional dissipation of dissipation. This version of the model could be viewed as an ensemble of flamelets which each experience a different dissipation rate, but also interact. In this respect there may also be parallels to multipleenvironment conditional PDF models [29]. Thankfully, all the other source terms, which would need models for cross dissipation and the net source of dissipation rate, were found to be negligible.

Future work could consider modelling of the dissipation of dissipation rate, or extension to other situations such as the situation with mixture-fraction stratification instead of thermal stratification 
or both types of fluctuations being simultaneously present.

\section{Acknowledgements}

This work was supported by the Australian Research Council (ARC). The research benefited from computational resources provided through the National Computational Merit Allocation Scheme, supported by the Australian Government. The computational facilities supporting this project included the Australian NCI National Facility, the partner share of the NCI facility provided by Intersect Australia Pty Ltd., the Pawsey Supercomputing Centre and the UNSW Faculty of Engineering. The work at Sandia National Laboratories was supported by the Combustion Energy Frontier Research Center, an Energy Frontier Research Center funded by the US Department of Energy (DOE), Office of Science, Office of Basic Energy Sciences under Award No. DE-SC0001198. Sandia is a multiprogram laboratory operated by Sandia Corporation, a Lockheed Martin Company, for the United States Department of Energy under contract DE-AC04-94AL85000. CSY was supported by Basic Science Research Program through the National Research Foundation of Korea (NRF) funded by the Ministry of Science, ICT and Future Planning (No. 2015R1A2A2A01007378).

\section{Appendix A. Supplementary material}

Supplementary material associated with this article can be found in the online version.

\section{References}

[1] J.E. Dec, Proc. Combust. Inst. 32 (2009) 2727-2742.

[2] J. Snyder, N. Dronniou, J. Dec, R. Hanson, SAE Int. J. Engines 5 (2011) 1669-1688.

[3] E.R. Hawkes, R. Sankaran, P.P. Pébay, J.H. Chen, Combust. Flame 145 (2006) 145-159.

[4] C.S. Yoo, T. Lu, J.H. Chen, C.K. Law, Combust. Flame 158 (2011) 1727-1741.

[5] C.S. Yoo, Z. Luo, T. Lu, H. Kim, J.H. Chen, Proc. Combust. Inst. 34 (2013) 2985-2993.

[6] R. Yu, X.S. Bai, Combust. Flame 160 (2013) 1706-1716.

[7] H.A. El-Asrag, Y. Ju, Combustion and Flame 161 (2014) 256-269.

[8] M. Talei, E.R. Hawkes, Proc. Combust. Inst. 35 (2015) 3027-3035. 
[9] F. Salehi, M. Talei, E.R. Hawkes, C.S. Yoo, T. Lucchini, G. D’Errico, S. Kook, Proc. Combust. Inst. 35 (2015) 3087-3095.

[10] A.Y. Klimenko, R.W. Bilger, Prog. Energ. Combust. Sci. 25 (1999) 595-687.

[11] F. Salehi, M. Talei, E.R. Hawkes, C.S. Yoo, T. Lucchini, G. D'Errico, S. Kook, Flow Turbul. Combust. (2015) 1-28.

[12] A. Kronenburg, R. Bilger, J. Kent, Proc. Combust. Inst. 27 (1998) 1097-1104.

[13] N. Swaminathan, R.W. Bilger, Phys. Fluids 11 (1999) 2679-2695.

[14] S.H. Kim, K.Y. Huh, Combust. Flame 138 (2004) 336-352.

[15] E. Richardson, C. Yoo, J. Chen, Proc. Combust. Inst. 32 (2009) 1695-1703.

[16] G. Paola, I. Kim, E. Mastorakos, Flow Turbul. Combust. 82 (2009) 455-475.

[17] A. Kronenburg, Phys. Fluids 16 (2004) 2640-2648.

[18] A. Kronenburg, A. Papoutsakis, Proc. Combust. Inst. 30 (2005) 759-766.

[19] J. Behzadi, M. Bolla, M. Talei, E. Hawkes, T. Lucchini, G. D'Errico, S. Kook, 10th APCC, Beijing, China, 2015, p. 323.

[20] R.W. Bilger, in: R.S.L. Lee, J.H. Whitelaw, T.S. Wung (Eds.), Aerothermodynamics in Combustors, Springer Verlag, Berlin, Heidelberg, 1991, p. 89.

[21] C.M. Cha, G. Kosály, H. Pitsch, Phys. Fluids 13 (2001) 3824-3834.

[22] A. El Sayed, C. Devaud, Proc. Combust. Inst. - Canadian Section, Université de Montreal, Quebec, 2009.

[23] S. Cao, T. Echekki, Combust. Flame 151 (2007) 120-141.

[24] G. Ruetsch, M. Maxey, Phys. Fluids 4 (1992) 2747-2760.

[25] A. Bhagatwala, J.H. Chen, T. Lu, Combust. Flame 161 (2014) 1826-1841.

[26] K.R. Sreenivasan, Flow Turbul. Combust. 72 (2004) 115-131. 
[27] E.E. O’Brien, T.L. Jiang, Phys. Fluids 3 (1991) 3121-3123.

[28] H. Pitsch, H. Barths, N. Peters, SAE Paper 962057, 1996.

[29] R.O. Fox, V. Raman, Phys. Fluids 16 (2004) 4551-4565. 


\section{List of Tables}

1 Physical parameters of the DNS and CMC cases. . . . . . . . . . . . . . 9

\section{List of Figures}

1 Initial temperature for case ethanol-5 with $T^{\prime}=40 \mathrm{~K} \ldots \ldots \ldots \ldots$

2 Modelling comparison; temporal evaluation of mean heat release rate in all cases. . . 9

3 Scatter plots of normalised $Y_{\alpha}-\left\langle\left. Y_{\alpha}\right|_{\xi}\right\rangle$ colour by $\log \left(N_{\theta}\right)$ versus normalised enthalpy for cases a) ethanol-5, b) octane-3, c) heptane-5, and d) heptane-9 . . . . . . . . . . . 10

4 Comparison of the left and right sides of doubly CMC equations, Eq. (8), for fuel mass fraction in case ethanol-5 with $T^{\prime}=40 \mathrm{~K} \ldots \ldots \ldots \ldots \ldots \ldots$

5 Evaluation of the different terms in DCMC equations, Eq. (8), for cases a) ethanol-5 with $T^{\prime}=40 \mathrm{~K}$ and b) octane- 3 with $T^{\prime}=60 \mathrm{~K}$. Test 1 , mixing in both $\theta$ - and $\phi$ spaces and their cross; Test 2 , mixing in both $\theta$ - and $\phi$ - spaces; Test 3 , only mixing

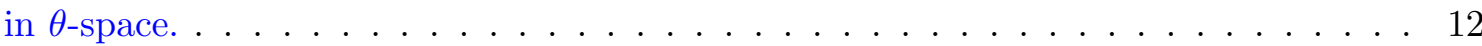

